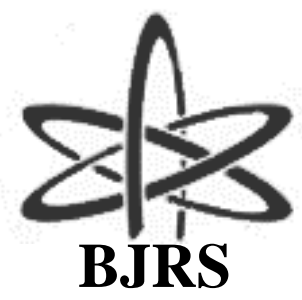

BRAZILIAN JOURNAL

$\mathrm{OF}$

RADIATION SCIENCES

08-01B (2020) 01-20

\title{
Medidas experimentais e simulação MCNPX para avaliar coeficiente de atenuação linear e mássico de catalisador ativado utilizado no processo de FCC.
}

\author{
Silva $^{a}$ V.H.F.F., Oliveira ${ }^{a}$ M.F.M., Dantas ${ }^{a}$ C. C ., Viana ${ }^{a}$ M.S., Resende Filho ${ }^{b}$ T.A., \\ Farias $^{a}$ W.A.W.A., Melo ${ }^{c}$ S. B., Lima ${ }^{a, b}$ F. R. A. \\ ${ }^{a}$ DEN/UFPE - Departamento de Energia Nuclear/Universidade Federal de Pernambuco, 50670-901, Recife-PE, Brasil \\ ${ }^{b}$ CRCN-NE - Centro Regional de Ciências Nucleares do Nordeste, 50730-120, Recife-PE, Brasil \\ ${ }^{c}$ CIN/UFPE - Centro de Informática/Universidade Federal de Pernambuco, 50.740-560, Recife-PE, Brasil
}

vitohugo1020@gmail.com

\section{RESUMO}

O Craqueamento Catalítico Fluido (FCC) é um processo utilizado na indústria petroquímica para converter algumas frações do petróleo que são mais pesadas em frações mais leves, como a gasolina e o GLP, por exemplo. A conversão ocorre devido à introdução de partículas sólidas denominadas catalisadores no interior de risers industriais de FCC. O catalisador aquecido entra em contato com gasóleo líquido na base do riser, promovendo a vaporização e o craqueamento dos vapores de hidrocarbonetos que compõem o gasóleo. Os parâmetros físicos e de fluxo da partícula, na maioria das vezes, são determinados por meio do uso de técnicas não intrusivas envolvendo a tomografia por transmissão gama, que possuem a vantagem de não pertubar o sistema. Este estudo teve por objetivo caracterizar a morfologia, composição química e densidade aparente do catalisador de FCC em estado ativado, bem como, avaliar o coeficiente de atenuação linear e mássico através de simulações no código MCNPX e irradiação experimental utilizando fontes radioativas de Césio-137 e Amerício-241 e comparar com as informações extraídas do XCOM do NIST. Os dados de saída das simulações e experimentos foram introduzidos na equação de Beer-lambert, aqui adaptada para a equação linear reduzida de uma reta, onde foi aplicado o coeficiente de determinação como fator de ajuste, que atestou o bom funcionamento do modelo proposto. 
Palavras-chave: Catalisador ativado, Irradiação, MCNPX, XCOM, Simulações.

\section{ABSTRACT}

Fluid Catalytic Cracking (FCC) is a procedure commonly applied on petrochemical industries in terms of converting dense amounts of oil into lighter fractions, such as gasoline and GLP. It occurs due to the introduction of solid-state particles, referred to as catalysts, inside industrial FCC riser. Once in contact with liquid diesel on the riser base, post-heat components assist with both vaporization and cracking of hydrocarbons. Physical parameters and particle flowing are generally determined throughout non-intrusive techniques involving Gamma-ray Transmission Tomography, considered an innovative advantage when it comes to system interference issues. Apart from an assessment on morphology and chemical composition as well as apparent density of activated-state FCC catalysts, this study aimed to further analysis of linear and mass attenuation coefficients through simulations with MCNPX code. In addition, irradiation experiments with Cesium-137 and Americium-241 took place at comparison approaches regarding previous-extracted information from the NIST XCOM. Output data were adapted into a reduced linear form of Beer-Lambert's equation in which a coefficient of determination were considered as an adjustment factor, therefore attesting a proper functioning of the proposed model.

Keywords: Activated catalyst, Irradiation, MCNPX, XCOM, Simulations.

\section{INTRODUÇÃO}

O Craqueamento Catalítico Fluido (Fluid Catalytic Cracking - FCC) é um processo utilizado na indústria petroquímica para a conversão do petróleo, através da ação de um catalisador, em combustíveis de baixo peso molecular e alto valor agregado. O processo de FCC está intrinsecamente relacionado com a fluidização, processo no qual um material na condição sólida, nesse caso o catalisador, é levado para a condição de fluido. Sendo assim, o FCC ocorre no chamado Leito Fluidizado Circulante (Circulating Fluidized Bed - CFB), composto basicamente por um reator denominado riser, um vaso de separação e uma coluna de retorno. O riser é um tubo vertical aonde justamente ocorrem as reações de FCC. No vaso de separação o catalisador é separado e conduzido à coluna de retorno para ser injetado novamente no riser. No tocante ao catalisador, o mesmo pode ser enquadrado, considerando o diâmetro de sua partícula $\left(\mathrm{d}_{\mathrm{p}}\right)$ e a densidade dela $\left(\rho_{\mathrm{p}}\right)$, na classificação Geldart constituída pelos grupos A, B, C e D. O catalisador analisado no presente estudo está enqua- 
drado no grupo A, composta por catalisadores cujas moléculas são finas $\left(\mathrm{d}_{\mathrm{p}}<100 \mu \mathrm{m}\right)$ e de baixa densidade $\left(\rho_{\mathrm{p}}<1400 \mathrm{~kg} / \mathrm{m}^{3}\right)[1,2,3,4$ e 5$]$.

Em termos técnicos, o estudo do FCC está dividido em duas linhas: a cinética e a fluidodinâmica. A primeira linha de pesquisa tem como foco a cinética das reações de FCC, enquanto a segunda tem como objeto de estudo o escoamento do fluxo multifásico contendo a fase sólida (catalisador). A impossibilidade de realizar experimentos diretamente na planta industrial solicita a construção de Unidades Piloto a Frio (UPF) para estudar a fluidodinâmica. A UPF, que é uma planta em escala reduzida, construída com material transparente, com o intuito de facilitar a visualização do escoamento bifásico, em temperatura ambiente, do tipo gás (ar) - sólido (catalisador). Nessas plantas são determinadas, a partir dos modelos básicos da fluidodinâmica e das medidas tomadas na UPF, parâmetros fluidodinâmicos: fração volumétrica de sólidos $\left(\varepsilon_{\mathrm{s}}\right)$, concentração de sólidos $\left(\mathrm{C}_{\mathrm{s}}\right)$, velocidade da fase sólida $\left(u_{s}\right)$ e velocidade de escorregamento ( $\left.v_{\text {slip}}\right)$. Geralmente estes parâmetros são obtidos através da técnica de gradiente de pressão. Porém, essa medição provoca perturbações no escoamento bifásico em estudo. Assim, as medidas de pressão acabam sendo uma técnica invasiva. Por isso, a utilização da medição de parâmetros por meio da técnica de transmissão gama tem se destacado por ser um método não intrusivo e eficaz para o estudo da fluidodinâmica gás-sólido em risers de FCC [6 e 7].

Parâmetros, como a $\varepsilon_{\mathrm{s}}$, só podem ser mensurados mediante o conhecimento prévio das características físico-químicas do catalisador, como, por exemplo, morfologia, composição química, densidade aparente $(\rho)$, coeficiente de atenuação linear $(\mu)$ e mássico $(\mu / \rho)$. Estes dois últimos, podem ser avaliados experimentalmente com a introdução de fontes monoenergéticas de radiação gama, que permitem obter valores precisos de $\mu$. Já o $\mu / \rho$ é calculado através da razão do $\mu$ pelo $\rho$ do catalisador, ou seja, neste último caso é necessário ter o conhecimento da densidade do material estudado.

$\mathrm{O} \mu$ também pode ser determinado por meio de simulações do transporte da radiação com uso de um código Monte Carlo (MC). O código Monte Carlo N-Particle eXtended (MCNPX), desenvolvido no Laboratório Nacional de Los Alamos, escrito em Fortran 90, é um código de propósito geral que se baseia no método estatístico de MC. Na versão $2.5 \mathrm{C}$, podem ser modelados os transportes individuais de partículas para ampla faixa de energia: fótons (1 keV-100 GeV), elétrons (1 
$\mathrm{keV}-1 \mathrm{GeV})$ e nêutrons $\left(10^{-11} \mathrm{MeV}\right.$ e $\left.20 \mathrm{MeV}\right)$. Além disso, podem ser transportados acoplados (nêutrons/fótons/elétrons) todos em geometria tridimensional e em sistema heterogêneo [8].

O presente estudo visa caracterizar a morfologia, composição química e densidade aparente do catalisador de FCC em estado ativado, bem como, avaliar o coeficiente de atenuação linear e mássico através de medidas experimentais em conjunto com simulações no código MCNPX, utilizando fontes de Césio-137 e Amerício-241, e comparar com as informações extraídas do software XCOM do banco de dados do Instituto Nacional de Normas e Tecnologia (National Institute of Standards and Technology - NIST) [9].

\section{MATERIAIS E MÉTODOS}

Este estudo foi dividido em duas partes, sendo a primeira correspondente ao procedimento experimental e a segunda envolvendo simulações Monte Carlo com o código computacional MCNPX e comparação de dados extraídos do NIST XCOM. A parte experimental consistiu na determinação da morfologia, composição química, coeficiente de atenuação linear $(\mu)$, densidade aparente $(\rho)$ e coeficiente de atenuação mássico $(\mu / \rho)$ do catalisador. Foram executadas simulações com o MCNPX na finalidade de determinar e comparar valores de $\mu$ e $\mu / \rho$ com os obtidos em experimentos. Por fim, foi necessário recorrer ao banco de dados do NIST para obter o $\mu_{\mathrm{c}} / \rho$ através de simulações no software XCOM.

\subsection{Morfologia e composição do catalisador em estado ativado}

A análise morfológica do catalisador foi realizada por Microscopia Eletrônica de Varredura (MEV), por um microscópio eletrônico da TESCAN, modelo VEJA 3SBH. Através de imagens, foi possível verificar alterações na morfologia dos grãos do catalisador no estado ativado. Acoplado ao equipamento de MEV, há um difratômetro de radiação X XRD 6000, da Shimadzu, que permitiu determinar a composição química da partícula, com o intuito de avaliar as possíveis modificações químicas que ocorreram no catalisador ativado, decorrente do processo de craqueamento que levam à sua desativação. 
Os valores da composição, obtidos com o equipamento de Difração de Raios X (DRX), foram inseridos no software XCOM do NIST [9] para a determinação do coeficiente de atenuação mássico, que foi utilizado como medida comparativa de referência para validar as informações extraídas das irradiações experimentais.

\subsection{Coeficiente de atenuação linear}

A relação entre os fótons transmitidos pelo material absorvedor $(I)$ e os fótons transmitidos sem a presença do mesmo $\left(I_{0}\right)$, que é dada pela lei de Beer-Lambert [10], aqui utilizada para um feixe monoenergético e expressa por:

$$
I=I_{0} e^{-\mu(Z, E) x}
$$

em que $I$ e $I_{0}\left[\mathrm{~W} / \mathrm{cm}^{2}\right]$, com $[\mathrm{W}]=[\mathrm{J} / \mathrm{s}]$, representam, respectivamente, as intensidades dos feixes da radiação transmitida e inicial, $\mu$ é o coeficiente de atenuação linear $\left[\mathrm{cm}^{-1}\right]$, específico para cada material, podendo variar com número atômico do meio $(Z)$ e com a energia da fonte radioativa (E), e a espessura do objeto absorvedor (x), dada em [cm].

As intensidades $I$ e $I_{0}$ (Equação 01), foram determinadas a partir da Tomografia Computadorizada de Raios Gama de $1^{\mathrm{a}}$ Geração (TCG- $1^{\mathrm{a}} \mathrm{G}$ ). O aparelho utilizado nas medidas experimentais pode ser visto na Figura 1. 


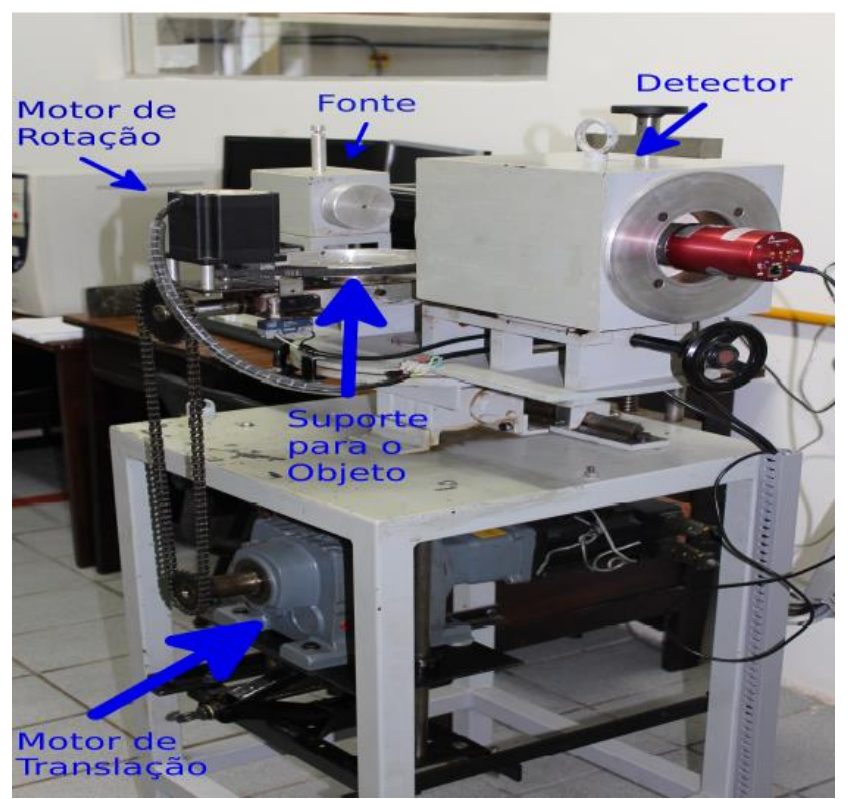

Figura 1: Tomógrafo Computadorizado de Raios Gama $\left(T G C-1{ }^{\circ} G\right)$.

Para determinar a fonte que permitirá a melhor inferência dos dados para as medidas tomográficas por transmissão gama na UPF, foram escolhidas dois radioisótopos, sendo um de baixa energia e outro de alta energia. Os dois emissores gama que satisfazem esse requisito são, respectivamente, o Am-241 e o Cs-137, cujas informações estão descritas na Tabela 1.

Tabela 1: Características das fontes de radiação gama utilizadas no presente trabalho.

\begin{tabular}{cccc}
\hline Fonte Radioativa & Fotopico (MeV) & Meia Vida (anos) & Atividade (Bq) \\
\hline Cs-137 & 0,662 & 30,1 & $3,7.10^{8}$ \\
Am-241 & 0,060 & 241 & $7,4.10^{9}$ \\
\hline
\end{tabular}

\subsection{Confecção e modelagem do recipiente de acrílico}

Para execução do procedimento experimental, foi confeccionado um recipiente retangular de acrílico com densidade do material de $1,19 \mathrm{~g} / \mathrm{cm}^{3}$ e as seguintes dimensões físicas: comprimento $(119,44 \pm 0,04) \mathrm{mm}$ e $k=2,43$, altura $(72,03 \pm 0,03) \mathrm{mm}, \operatorname{com} k=2,18$ e largura $(94,42 \pm 0,03) \mathrm{mm}$, com $k=2,18$; sendo $k$ o fator de abrangência, utilizado para calcular a incerteza expandida, com valor situado entre 2 e 3 , e que está relacionado com a determinação do intervalo de confiança de $95 \%$, a partir da distribuição de $t$-Student. O catalisador em estado ativado, cuja partícula é perten- 
cente ao grupo A da classificação Geldart, foi inserido no interior do recipiente especificado. Para obter uma comparação confiável entre simulação e experimento, um fantoma geométrico com as mesmas dimensões físicas e composição química do utilizado nas medidas experimentais, foi modelado em um arquivo de entrada MCNPX, também chamado de arquivo input. O input MCNPX é um arquivo de texto (txt.) formado por 4 blocos de dados: o Titulo (opcional), o Cartão de Células, o Cartão de Superfície e o Cartão de Dados. Nesse arquivo é possível especificar: tipo de fonte, de detector, configuração da geometria e condições gerais do sistema desejado, características do espectro de energia da fonte radioativa, bem como, o meio que a radiação irá interagir e definição da geometria do detector desejado [11].

O recipiente experimental e virtual MCNPX, sem e com catalisador, pode ser observado nas Figuras 2(a) e 2(b) e Figuras 3(a) 3(b), respectivamente.

(a) Sem catalisador

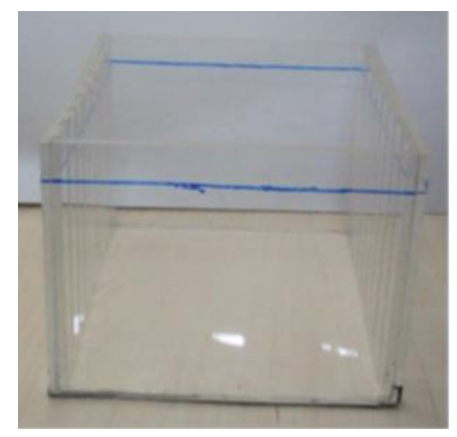

Figura 2: Recipiente experimental sem e com catalisador. (b) Com catalisador

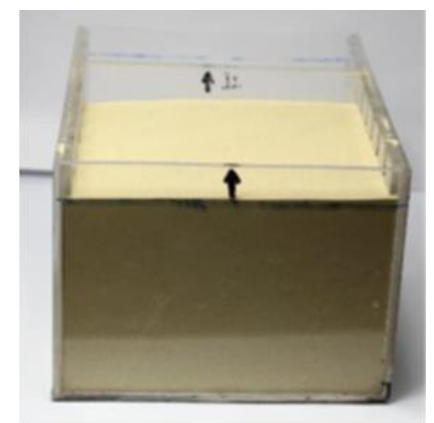

(a) Sem catalisador

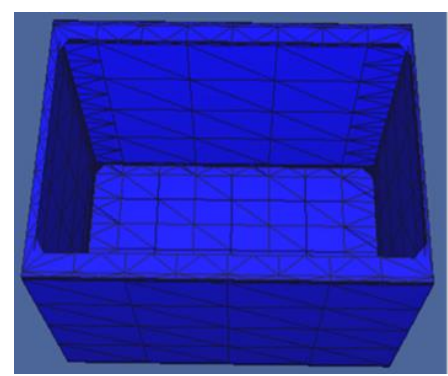

(b) Com catalisador

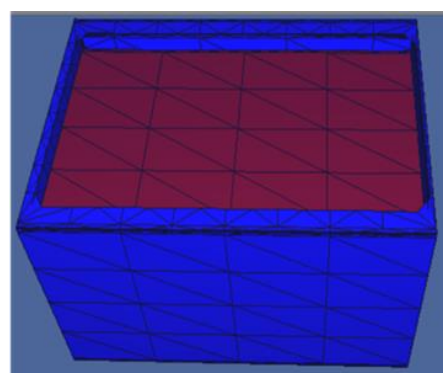

Figura 3: Recipiente virtual MCNPX sem e com catalisador. 


\subsection{Arranjo experimental e no código MCNPX}

No arranjo experimental, inicialmente, foi necessário realizar testes de determinação do posicionamento do recipiente de acrílico para que as medidas fossem adquiridas em condições de repetitividade. Sendo assim, o recipiente foi posicionado de forma que seu centro geométrico coincidiu com a origem do eixo de coordenadas $x$ e $y$ do tomógrafo.

Para simular as condições reais de irradiação no código MCNPX, também foi construído um tomógrafo com as mesmas características geométricas, físicas e químicas do TCG- $1^{\mathrm{a}} \mathrm{G}$ experimental (Figura 1). Foram introduzidas, através de comandos e funcionalidades específicas do input MCNPX [11], duas fontes gama caracterizadas pelo espectro de distribuição de energia discreto (0,060 MeV e 0,662 MeV), colimador de chumbo com orifício de 1,25 mm de diâmetro para entrada do feixe emergente do objeto irradiado e detector cintilador (NaI-Tl). O recipiente virtual foi posicionado da mesma maneira da apresentada no arranjo experimental, tendo o seu centro geométrico distante $11,2 \mathrm{~cm}$ e 17,2 cm, respectivamente, da fonte e detector. Na Figura 4 é visto o posicionamento do recipiente no tomógrafo experimental (esquerda) e no tomógrafo MCNPX (direita).
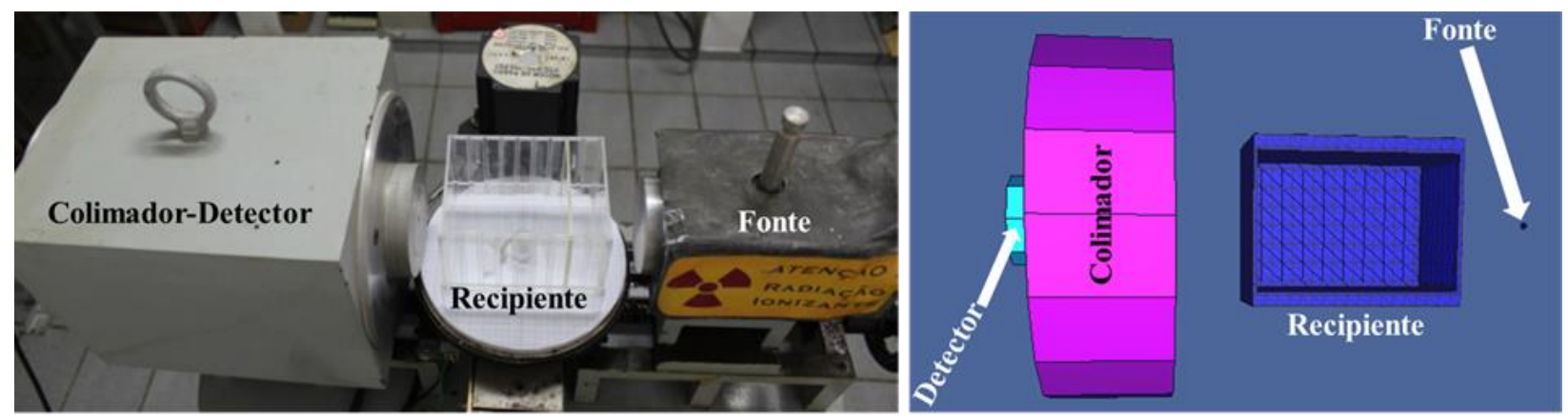

Figura 4: Posicionamento do recipiente vazio no tomógrafo experimental (esquerda) e virtual MCNPX (direita).

O recipiente foi construído com divisórias fixas, na finalidade de obter valores de contagens distintos, referentes a atenuação do feixe pelas diferentes camadas de catalisador. Desta forma, o $\mu$ é dado em função do $I$ e $x$, sendo $I$ o valor de intensidade da radiação que interagiu com o volume sensível do detector e $x$ a espessura decorrente das divisões criadas.

A variação do espaçamento entre as divisórias fixas, $x_{j}$, foi obtida por uma progressão aritmética, representada por: 


$$
x_{i}=x_{i-1}+x_{0} \text { para } j=1,2,3, \ldots, N-1, N .
$$

sendo $x_{0}$ a espessura do compartimento com catalisador na primeira posição $(12,75 \pm 0,11 \mathrm{~mm}) \mathrm{e}$ $\mathrm{N}=8$ é a quantidade de divisórias criadas.

\subsection{Irradiação do recipiente de acrílico vazio e com catalisador de FCC}

As irradiações foram exexutadas no Laboratório de Fluidodinâmica e Ensaios Não-Destrutivos do Departamento de Energia Nuclear da Universidade Federal de Pernambuco (DEN/UFPE). E as simulações no código MCNPX foram realizadas em um conjunto de computadores, denominado clusters, presentes no Laboratório de Física de Solos do mesmo departamento.

No arranjo experimental, para a captação dos fótons gama, foi utilizado um detector acoplado a uma fotomultiplicadora ligada a um Analisador Multicanal - MCA modelo Osprey. Conforme apresentado na Figura 1, o sistema mecânico do tomógrafo é movimentado por motores de passo, com coordenadas $(x, y)$, onde $x$ representa o passo de translação ou ângulo de rotação e $y$ corresponde a medida de transmissão gama. $\mathrm{O}$ programa de controle do sistema $\mathrm{TCG}-1^{\mathrm{a}} \mathrm{G}$ foi desenvolvido através do software LabVIEW [1], o qual interage com o sistema de detecção e movimento da mesa.

As simulações MCNPX foram realizadas com energias de 0,060 MeV (Am-241) e 0,662 MeV (Cs-137). Em ambos os casos, o código aplicou automaticamente o tratamento físico detalhado, que leva em consideração o espalhamento Thomson e os fótons fluorescentes. $\mathrm{O}$ transporte de elétrons foi desconsiderado, sendo computado apenas o número de fótons primários que incidiam sobre a superfície de detecção. Como uma medida da boa qualidade dos resultados obtidos nas simulações, foi utilizado o erro relativo $(\mathrm{R})$, definido pelo desvio padrão estimado da média $\left(S_{\bar{x}}\right)$ dividido pela média estimada $(\bar{x})$. O erro ralativo é proporcional a $1 / \mathrm{N}^{1 / 2}$, onde $\mathrm{N}$ é o número de histórias [11]. Foram realizadas 20 simulações, sendo 10 para fótons de $0,060 \mathrm{MeV}$ e outras 10 para fótons de $0,662 \mathrm{MeV}$, com todas simulações tendo $\mathrm{N}=10^{7}$ histórias.

O arranjo experimental e o arranjo no código MCNPX são vistos, respectivamente, nas Figuras 5 e 6, onde observa-se a variação da espessura das camadas de catalisador no recipiente de acrílico, de forma a indicar a variação do volume de preenchimento da caixa, conforme a Equação 02, para o primeira e segunda camada, nesta ordem. 
a) Primeiro compartimento

b) Segundo compartimento

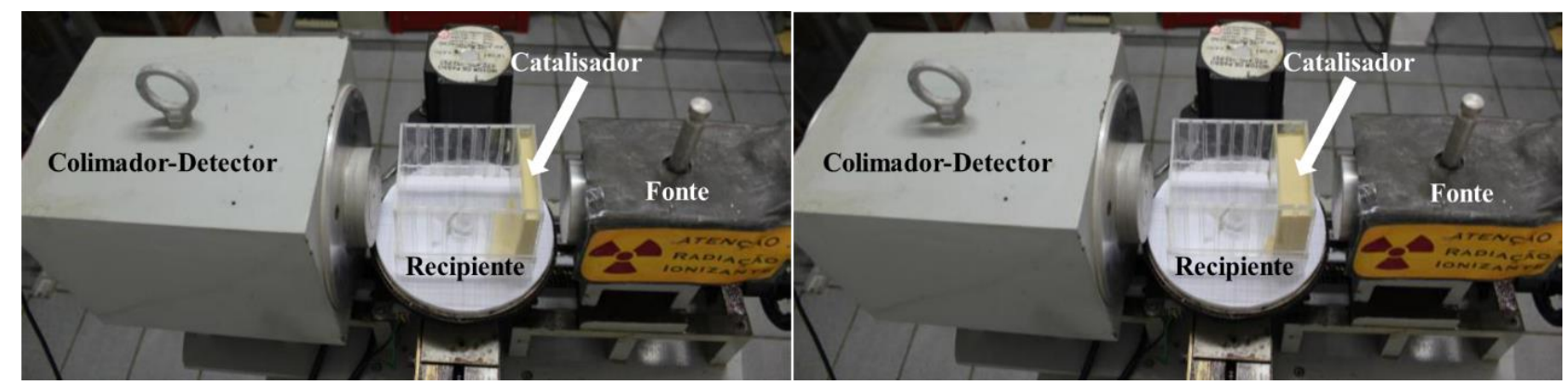

Figura 5: Arranjo experimental com camadas de catalisador de FCC no tomógrafo gama.

a) Primeiro compartimento

b) Segundo compartimento
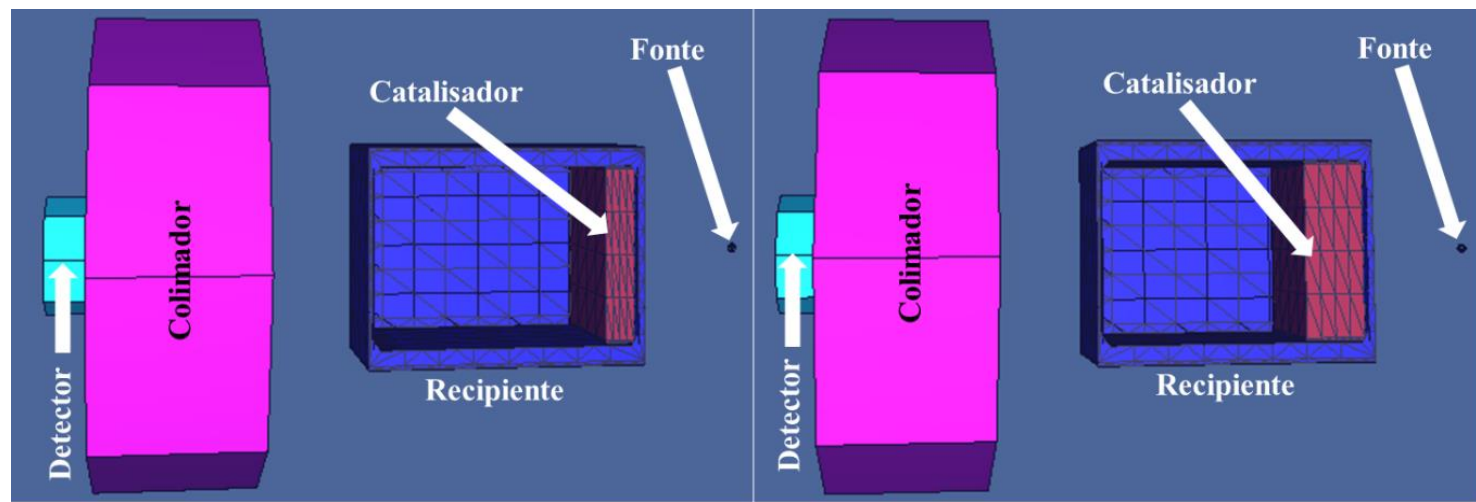

Figura 6: Geometria do sistema com camadas de catalisador de FCC no MCNPX.

Os parâmetros de varredura TCG $-1^{\circ} \mathrm{G}$ utilizados no experimento foram: posição de leitura de referência e tempo de contagem, cujos valores utilizados estão apresentados na Tabela 2.

Tabela 2: Parâmetros do sistema de controle do TCG - $1^{\mathrm{a}} \mathrm{G}$ manual.

\begin{tabular}{ccc}
\hline Variável de processo & Valor & Unidade \\
\hline Posição de leitura de referência, $\mathrm{x}_{\mathrm{ref}}$ & 91 & $\mathrm{~mm}$ (milímetros) \\
Tempo de contagem, $\mathrm{t}_{\mathrm{c}}$ & 180 & $\mathrm{~s}$ (segundos) \\
\hline
\end{tabular}


No tomografo experimental foram realizadas 5 repetições para cada compartimento nas condições com e sem catalisador, totalizando 100 irradiações, dos quais 10 ensaios para caixa de acrílico nas condições de vazio.

\subsection{Modelagem matemática da atenuação de raios gama}

O sistema analisado é formado pelos materiais que compõem a caixa de acrílico e pelo catalisador. Portanto, reescrevendo a Equação 01, tem-se que a intensidade do feixe que atravessa a caixa de acrílico com catalisador, $I_{c}$ (feixe de raios gama transmitido), poder ser expresso:

$$
I_{c}=I_{v} e^{-\mu_{0} x_{c}}
$$

em que $\mu_{\varepsilon}$ é o coeficiente do de atenuação linear do catalisador a ser analisado $\left[\mathrm{cm}^{-1}\right], x_{\varepsilon}$ é a espessura da trajetória composta por catalisador [cm], e $I_{v}$ é a intensidade do feixe que atravessa a caixa de acrílico vazia, determinada por:

$$
I_{v}=I_{0} e^{-\mu_{a} x_{a}}
$$

sendo, $\mu_{a}$ é o coeficiente de atenuação linear do material de acrílico $\left[\mathrm{cm}^{-1}\right] ; I_{0}$ é a intensidade da radiação inicial, e $x_{a}$ é a espessura de acrílico percorrida pela radiação $\left(x_{a}=2 x\right)$.

Nos dados de saída das simulações e experimento, ao invés de utilizar as informações referentes a intensidade do feixe transmitido, $I_{v}$, definido pela Equação 04, foi levado em consideração as informações de atenuação no recipiente de acrílico nas mesmas condições, mas sem catalisador.

Modificando a Equação 03 e inserindo logaritmos neperianos em ambos os lados, obtém-se a equação da atenuação de raios gama para o catalisador, definida por:

$$
\ln \left(I_{v}\right)=\ln \left(I_{c}\right)+\mu_{c} x_{c}
$$

sendo $I_{c}$ e $I_{v}$ as intensidades do feixe que atravessam a caixa de acrílico com e sem catalisador, respectivamente, $\mu_{\varepsilon}$ corresponde ao coeficiente de atenuação linear do catalisador a ser analisado $\left[\mathrm{cm}^{-1}\right]$, e $x_{\varepsilon}$ a espessura da trajetória com catalisador $[\mathrm{cm}]$.

Reescrevendo a Equação 05, na forma da equação linear reduzida de uma reta, tem-se: 


$$
y_{\text {mod }}=b_{0}+b_{1} x
$$

assim, a atenuação da radiação gama representada por $y_{\bmod }=\ln \left(l_{c} / l_{v}\right), b_{0}=0$ e $b_{1}=\mu_{\varepsilon}$ o coeficiente de atenuação linear do catalisador.

Como visto na Equação 05, o coeficiente de atenuação linear pode ser estimado, através de uma equação linear, para os diferentes valores de intensidade relativa obtidos a partir da variação de espessuras criadas na caixa de acrílico. Para tal, foi utilizado o método de Mínimos Quadrados Linear (MQL), que corresponde a um ajuste baseado na minimização dos erros residuais entre os pontos da curva e os dados determinados nas simulações e experimentos [2].

Por meio do método MQL, que pode ser representado pela Equação 06, foi obtida a equação da reta que melhor representa os dados de atenuação adquiridos, com a finalidade de determinar os parâmetros $b_{1}$, que representa o coeficiente de atenuação linear do catalisador de FCC em estado ativiado.

\subsection{Densidade aparente do catalisador}

A densidade aparente, $\rho$, para o catalisador ativado foi calculada pela seguinte expressão:

$$
\rho=\frac{m}{V}
$$

em que $m$ é a massa de catalisador [g] que ocupou o volume $\left[\mathrm{cm}^{3}\right], V$, do cilindro de acrílico utilizado para dispor o catalisador na obtenção das medidas de massa.

O tubo cilíndrico de acrílico possui as seguintes dimensões físicas: 1,6042 $\pm 0,0067 \mathrm{~cm}$ de diâmetro interno, com $k=2,52$ e 10,5192 $\pm 0,0046 \mathrm{~cm}$ de altura interna, com $k=2,28$. Na Figura 7 pode-se observar o recipiente de catalisador sem e com material.
(a) Sem material
(b) Com material 

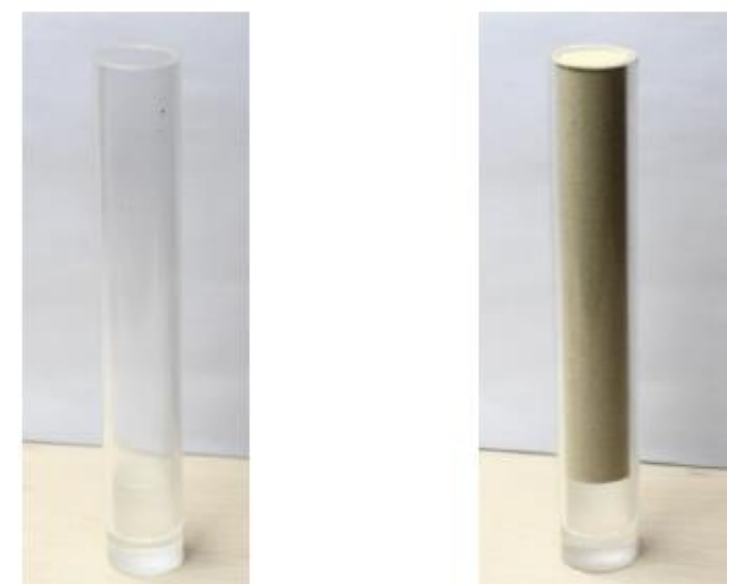

Figura 7: Recipiente de catalisador de FCC.

A massa do catalisador foi medida com uma balança analítica Shimadzu, modelo AUY220. Primeiramente foram feitas três medidas do recipiente vazio, a cada medida a balança foi zerada para que não houvesse interferência das medidas anteriores. Após isso, foi inserido catalisador ativado até que o recipiente fosse completamente preenchido. A metodologia utilizada para o recipiente vazio se repetiu para o recipiente com o catalisador.

\subsection{Coeficiente de atenuação mássico}

Além das desvantagens citadas anteriormente, a utilização do coeficiente de atenuação linear apresenta uma outra desvantagem referente à variação do valor com o estado físico do material. A partir do cálculo da densidade aparente e da determinação do $\mu_{\varepsilon}$ experimental e simulado, foi possível obter o coeficiente de atenuação mássico para o catalisador $\left[\mathrm{cm}^{2} / \mathrm{g}\right]$.

Os valores do coeficiente de atenuação mássico do catalisador de FCC, foram determinados por meio da seguinte equação:

$$
\mu_{m}=\frac{\mu_{c}}{\rho}
$$

em que $\mu_{\epsilon}$ e $\rho$ são, respectivamente, o coeficiente de atenuação linear e densidade aparente do catalisador em estado ativado. 
Além disso, as informações referentes à composição química e fração de massa para cada elemento que compõe o catalisador possibilitaram a obtenção do $\mu_{c} / \rho$ por meio de simulações no software XCOM do banco de dados do NIST [9]. Assim, foi possível comparar o $\mu_{\mathrm{c}}$ e o $\mu_{\mathrm{c}} / \rho$ a partir dos dados extraídos das irradiações experimentais, simulações no código MCNPX e XCOM.

\section{RESULTADOS E DISCUSSÃO}

Os resultados alcançados neste trabalho estão descritos e separados por tópicos de acordo com as etapas realizadas e metodologias aplicadas, que são: morfologia e composição do catalisador em estado ativado, coeficiente de atenuação linear, densidade aparente e coeficiente de atenuação mássico.

\subsection{Morfologia e composição do catalisador em estado ativado}

$\mathrm{Na}$ análise realizada com Microscopia eletrônica de varredura (MEV), foi possível observar a estrutura morfológica do catalisador, conforme pode ser observada na Figura 8. A utilização da MEV permitiu visualizar uma distribuição de poros nas partículas, chamados sítios ativos. Estes sítios ativos são responsáveis pelas reações de quebra das moléculas de hidrocarboneto no processo de Craqueamento Catalítico Fluido (FCC) do petróleo na indústria petroquímica.

(a)

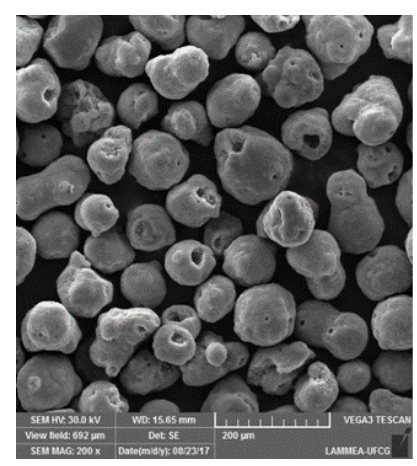

(b)

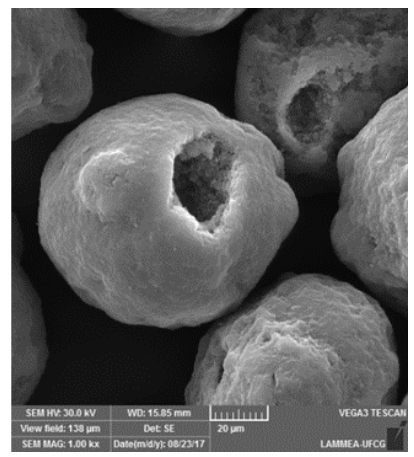

Figura 8: Microscopia eletrônica de varredura (MEV) do catalisador de FCC ativado com aumento de 200X, (a) e $1 \times 10^{3} \mathrm{X},(b)$. 
Através da difração de raios $\mathrm{X}$, foram obtidos os espectros correspondentes à composição química do catalisador ativado, conforme apresentado na Figura 9.

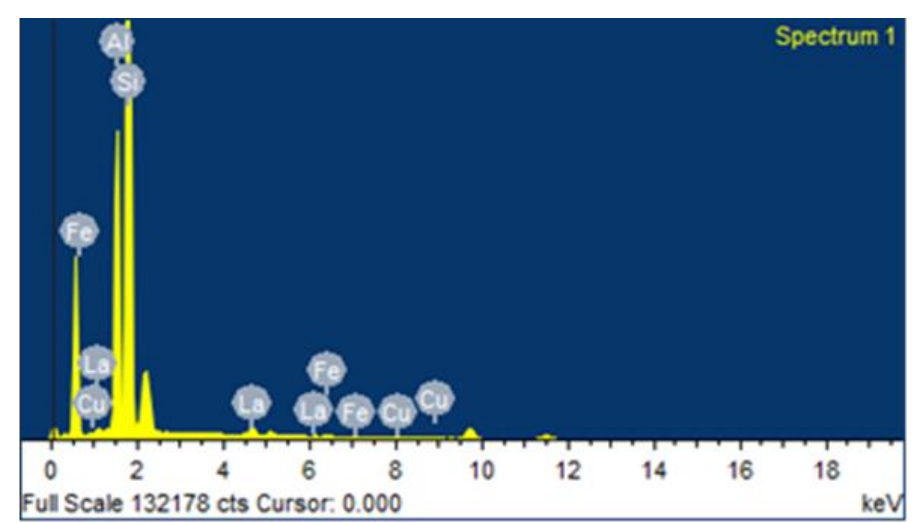

Figura 9: Espectro da difração de raios $X(D R X)$ de amostra de catalisadores ativado.

A análise química observada no espectro da Figura 9, mostra o catalisador em estado ativado com 95,662\% da composição como sendo de dióxido de silício $\left(\mathrm{SiO}_{2}\right)$ e óxido de alumínio $\left(\mathrm{Al}_{2} \mathrm{O}_{3}\right)$. As substâncias óxido de lantânio $\left(\mathrm{La}_{2} \mathrm{O}_{3}\right)$, pentóxido de fósforo $\left(\mathrm{P}_{2} \mathrm{O}_{5}\right)$ e óxido de ferro $\left(\mathrm{Fe}_{2} \mathrm{O}_{3}\right)$ correspondem a 3,693\% da massa total (Tabela 3). Por se tratar de catalisador em estado ativado, não possui grandes quantidades de metais incorporados na catálise $\left(\mathrm{Fe}_{2} \mathrm{O}_{3}, \mathrm{~V}_{2} \mathrm{O}_{3}\right.$ e NiO) [6].

Tabela 3: Composição mássica dos catalisadores de FCC nas condições ativado utilizando a técnica de DRX.

\begin{tabular}{cc}
\hline Compostos & Fração de massa (\%) \\
\hline $\mathrm{SiO}_{2}$ & 66,980 \\
$\mathrm{Al}_{2} \mathrm{O}_{3}$ & 28,682 \\
$\mathrm{La}_{2} \mathrm{O}_{3}$ & 1,557 \\
$\mathrm{P}_{2} \mathrm{O}_{5}$ & 1,438 \\
$\mathrm{Fe}_{2} \mathrm{O}_{3}$ & 0,698 \\
Outros & 0,65 \\
\hline
\end{tabular}




\subsection{Coeficiente de atenuação linear}

Conforme o modelo apresentado na Equação 05, os valores de atenuação do feixe de radiação em função das variações de espessura do catalisador na caixa de acrílico, tanto para dados experimentais (pontos) quanto para dados extraídos das simulações MCNPX (reta), são mostrados nos gráficos de dispersão, ajustados pelo método MQL para o catalisador ativado (Figuras 10 e 11).

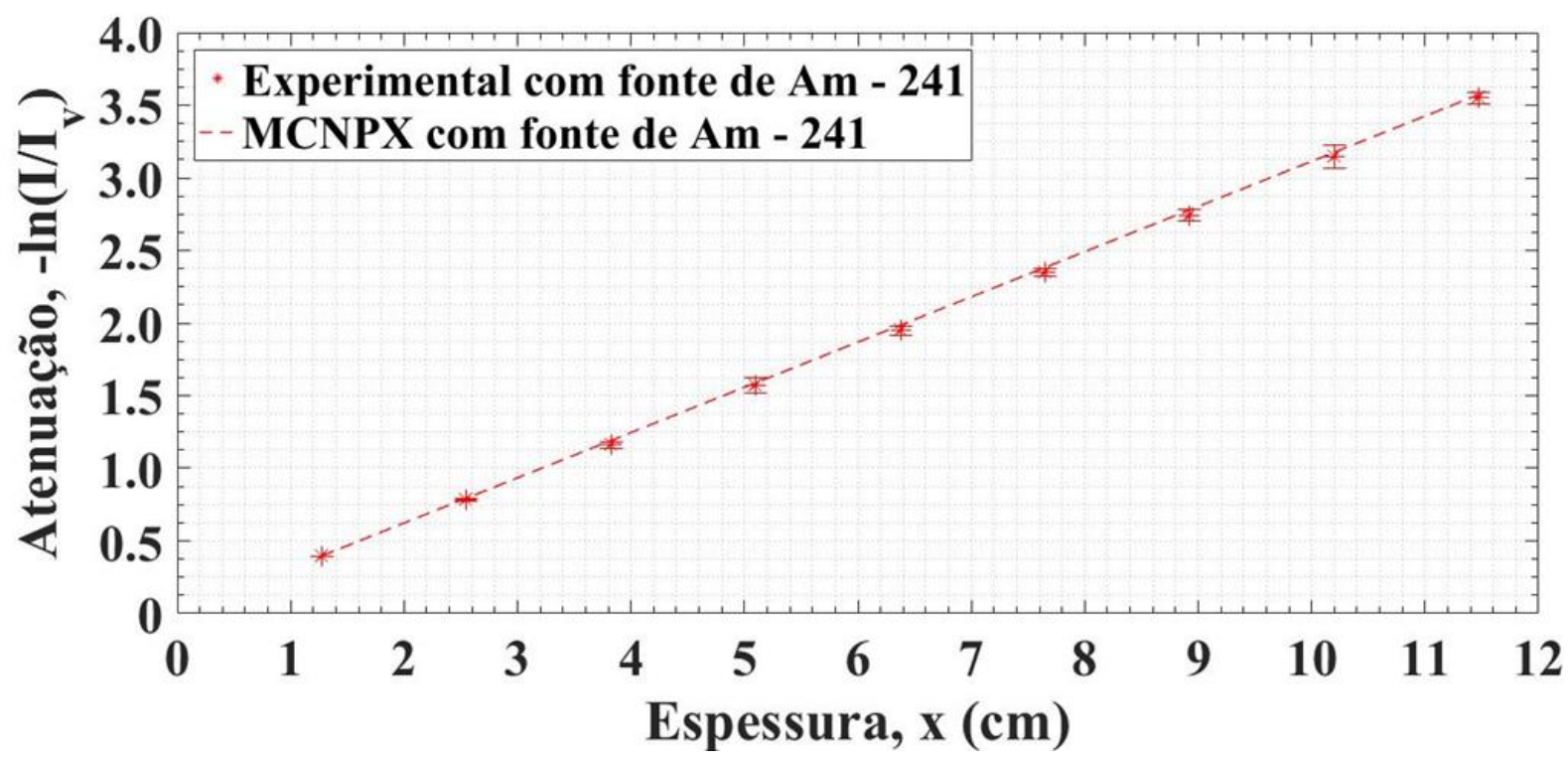

Figura 10: Comparação da atenuação dos fótons gama para o catalisador ativado, utilizando fontes de Am-241. 


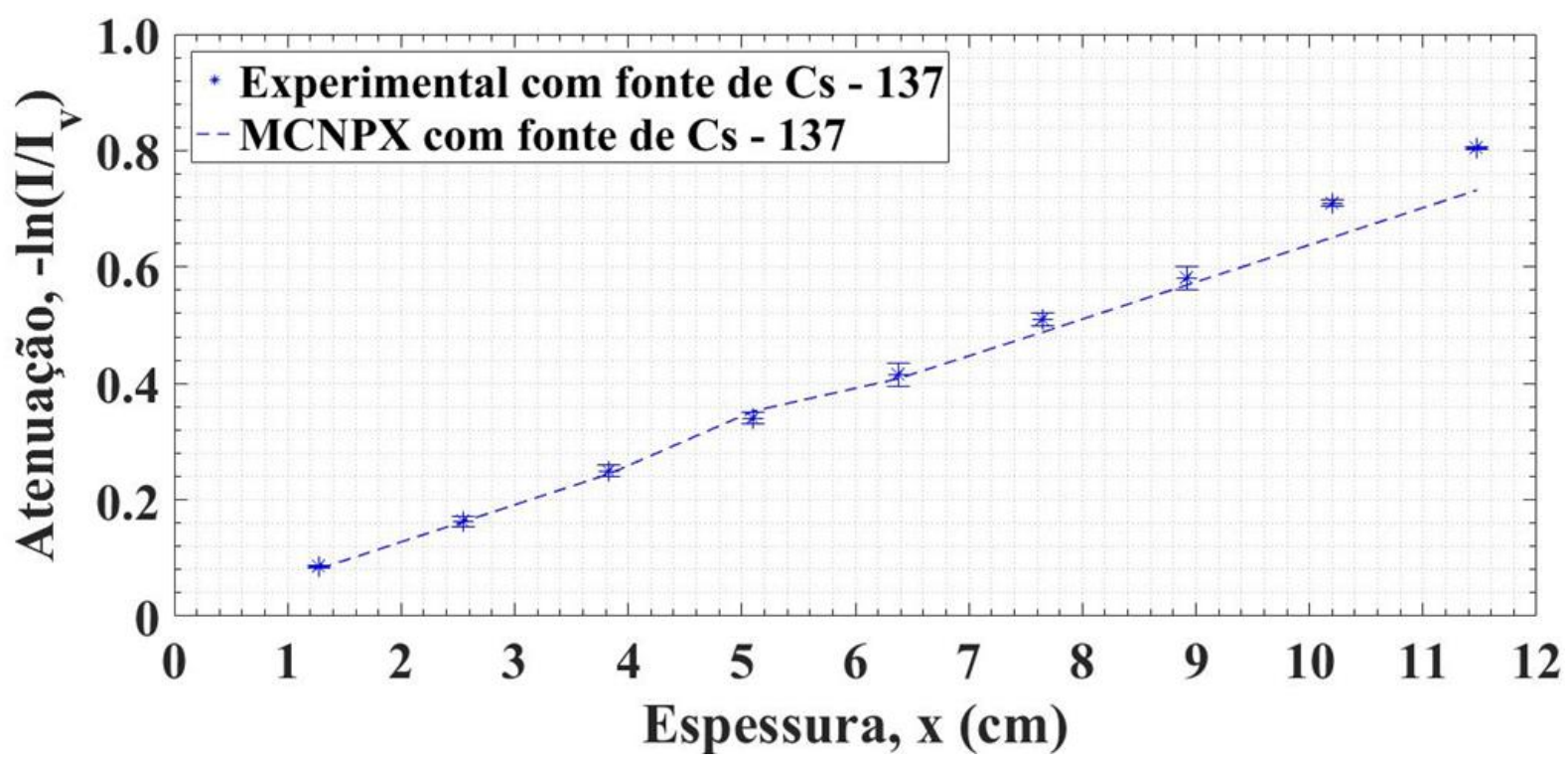

Figura 11: Comparação da atenuação dos fótons gama para o catalisador ativado, utilizando fontes de Cs-137.

A qualidade do ajuste demonstrado nas Figuras 10 e 11 para os dados MCNPX, foi quantificada pelo coeficiente de determinação $\left(\mathrm{r}^{2}\right)$. O valor de $\mathrm{r}^{2}$ encontrado foi de 0,9999 e 0,9999 , para o catalisador irradiado com fótons de $0,060 \mathrm{MeV}$ e $0,662 \mathrm{MeV}$, nesta ordem, indicando que a reta de regressão encontrada é capaz de explicar $99 \%$ dos dados. No arranjo experimental, os valores de $\mathrm{r}^{2}$, obtidos através da irradiação do catalisador ativado por fontes de Am-241 e Cs-137, foram de 0,9999 e 0,9967, respectivamente. Assim, a reta de regressão encontrada no experimento também foi capaz de explicar $99 \%$ dos dados da população, ou seja, em ambos os casos o valor de $\mathrm{r}^{2}$ é muito próximo, atestando o bom funcionamento do modelo proposto.

A comparação entre os valores de $b_{1}$, que representam as médias de $\mu_{\mathrm{c}}$ obtidos com as simulações MCNPX e irradiações experimentais, são dados na Tabela 4.

Tabela 4: Comparação dos valores do coeficiente de atenuação linear, utilizando fonte de Am-241 e Cs-137, para o catalisador ativado.

\begin{tabular}{ccc}
\hline Fonte & \multicolumn{2}{c}{ Coeficiente de Atenuação Linear $\left(\mathbf{c m}^{-\mathbf{1}}\right)$} \\
\cline { 2 - 3 } Radioativa & Média das Simulações MCNPX & Média Experimental \\
Am-241 & $0,3115 \pm 0,0003$ & $0,3159 \pm 0,0092$ \\
Cs-137 & $0,0638 \pm 0,0001$ & $0,0706 \pm 0,0044$ \\
\hline
\end{tabular}


Os valores de intervalo de confiança indicam que há $95 \%$ de probabilidade de que o valor verdadeiro do parâmetro que representa a população, $b_{1}$, estejam entre este intervalo. Segundo os valores de intervalo de confiança listados na Tabela 4, pode-se concluir que $b_{1}$ é um parâmetro significativo por não haver possibilidade de $b_{1}=0$, sendo assim, $\mu$ pode ser representado por $b_{1}$ em todos os casos.

Através de cálculos analíticos, o erro relativo entre a média simulada e a média experimental é de 1,41\% para fonte de Am-241 (0,060 MeV). Considerando a fonte de Cs-137 (0,662 MeV), o valor do erro relativo é de $3,76 \%$.

\subsection{Densidade aparente do catalisador}

O valor de densidade aparente $(\rho)$ do catalisador ativado, associado à incerteza expandida, foi de $0,8332( \pm 0,0031) \mathrm{g} / \mathrm{cm}^{3}, \operatorname{com} k=2,43$ para a medida. Este valor é muito próximo ao encontrado na literatura, onde a densidade aparente do catalisador, apresentada por outros autores, foi de 0,76 $\mathrm{g} / \mathrm{cm}^{3}$ e $0,79 \mathrm{~g} / \mathrm{cm}^{3}[12]$.

\subsection{Coeficiente de atenuação mássico}

Para um catalisador com partícula pertencente ao grupo A de Geldart [1], a densidade utilizada foi a densidade aparente $\left[\mathrm{g} / \mathrm{cm}^{3}\right]$. Assim, a divisão de $\mu_{\mathrm{c}}$ pelo valor de $\rho$ do material, possibilitou a determinação do coeficiente de atenuação mássico para o catalisador estudado $\left(\mu_{\mathrm{c}} / \rho\right)$. Na Tabela 5, é apresentada a comparação entre o $\mu_{\mathrm{c}} / \rho$ extraído em simulações XCOM do banco de dados do NIST [9] com o valor médio experimental e o obtido na média das simulações no código MCNPX.

Tabela 5: Comparação dos valores do coeficiente de atenuação mássico, utilizando fonte de Am241 e Cs-137, para o catalisador ativado.

\begin{tabular}{cccc}
\hline \multirow{2}{*}{ Fonte } & \multicolumn{3}{c}{ Coeficiente de Atenuação Mássico $\left(\mathbf{c m}^{2} / \mathbf{g}\right)$} \\
\cline { 2 - 4 } Radioativa & Média das & NIST & Média \\
& Simulações MCNPX & XCOM & Experimental \\
Am-241 & $0,3738 \pm 0,0002$ & 0,3770 & $0,3791 \pm 0,0047$ \\
Cs-137 & $0,0765 \pm 0,0001$ & 0,0704 & $0,0847 \pm 0,0053$ \\
\hline
\end{tabular}


O erro relativo para o coeficiente de atenuação mássico do catalisador de FCC encontrado para o valor simulado para fótons de $0,060 \mathrm{MeV}$ e o de referência no XCOM é de $0,86 \%$. O erro relativo entre a média da simulação e experimental é de 1,4\% para a mesma energia. No caso dos valores do erro para os procedimentos envolvendo a fonte de Cs-137 (0,662 MeV), é de 7,97\% para simulação e NIST. E entre simulação e experimento é de 3,79\%.

A comparação entre os erros, mostra uma diferença praticamente insignificante entre os valores obtidos por simulações, experimento e no XCOM. No entanto, os resultados da análise comparativa de erro para fonte de Cs-137, tanto para o $\mu_{\mathrm{c}}$ quanto para $\mu_{\mathrm{c}} / \rho$, foram superiores aos obtidos para fonte de Am-241, que pode ser explicável devido a baixa densidade dos materiais e elevada energia do feixe oriundo da fonte de Cs-137.

\section{CONCLUSÕES}

No tocante à morfologia e composição do catalisador em estado ativado, a combinação de duas técnicas experimentais foi de suma importância, pois ambas se complementam. A técnica de Microscopia eletrônica de varredura (MEV) permite a observação dos sítios ativos, os responsáveis pelas quebras das moléculas de hidrocarboneto no processo de FCC; enquanto a medida experimental de difração por raios $\mathrm{X}(\mathrm{DRX})$ possibilitou determinar a composição mássica do catalisador. Assim, a primeira técnica trouxe uma visão preliminar para a escolha ou não da utilização do catalisador, enquanto a outra foi essencial para o cálculo da densidade aparente $(\rho)$ e, consequentemente, do coeficiente de atenuação mássico $\left(\mu_{m}\right)$. Porém, para o cálculo desse último, é fundamental o conhecimento do coeficiente de atenuação linear $\left(\mu_{c}\right)$, uma das etapas realizadas. A determinação de $\mu$ também permitiu a escolha da fonte radioativa mais apropriada. E a partir da comparação entre as medidas com duas fontes radioativas, foi mostrado que a fonte de Am-241, de energia menor que a do Cs-137, resulta num erro relativo menor em relação ao valor de referência do NIST XCOM. Assim, para a composição do catalisador analisado e as condições do sistema experimental considerado, a fonte de Am-241 é mais apropriada. A utilização das medidas experimentais e de simulações com o código MCNPX possibilitaram a obtenção dos valores médios do coeficiente de atenuação mássico $\left(\mu_{m, m e ́ d i o}\right)$ para o catalisador ativado, parâmetro básico para análise fluidodinâmica através de medidas de radiação. O modelo aplicado mostrou resultados 
satisfatórios para as fontes radioativas, cujos respectivos resultados foram: $\left(\mu_{m, m e ́ d i o(\mathrm{MCNPX})}=0,3738\right.$

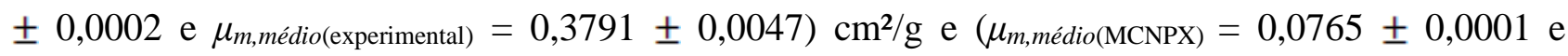
$\left.\mu_{m \text {,médio(MCNPX) }}=0,0847 \pm 0,0053\right) \mathrm{cm}^{2} / \mathrm{g}$, com valores de erro relativo entre valor experimental e o gerado por simulação MCNPX abaixo de 4,0 \%, mostrando boa consistência entre ambos. Isto faz com que a técnica de simulação por MCNPX possa ser utlizada com boa confiabilidade para efeitos de comparação entre valores e, principalmente, diante da inviabilidade da tomada de medidas através da técnica experimental por meio de transmissão gama.

\section{REFERÊNCIAS}

[1] GELDART, D. Types of Gas Fluidization. Powder Technology, v. 7, p. 285 - 292, 1973;

[2] BASU, P. Circulating Fluidized Bed Boilers: Design, Operation and Maintenance. Springer, p. 343, 2015;

[3] MOURA, A. E. Controle da injeção de catalisador em uma unidade tipo FCC a frio. 88 f. 2015. Dissertação (Mestrado em Tecnologias Energéticas e Nucleares) - Departamento de Energia Nuclear, Universidade Federal de Pernambuco, Recife;

[4] YATES, J. G.; LETTIERI, P. Fluidized-Bed Reactors: Processes and Operating Conditions. Springer, p. 1, 2016;

[5] OLIVEIRA, M. F. M. Modelagem do transporte de sólidos em regime denso em uma unidade piloto a frio por medição de transmissão gama. 104 f. 2018. Dissertação (Mestrado em Tecnologias Energéticas e Nucleares) - Departamento de Energia Nuclear, Universidade Federal de Pernambuco, Recife;

[6] LIMA FILHO, H. J. B.; BRITO, M. F. P.; BENACHOUR, M.; SANTOS, V. A.; DANTAS, C.C.; Validação experimental de simulações cfd de um leito fluidizado circulante gás-sólido tipo riser. XX Congresso Brasileiro de Engenharia Química, 2014;

[7] BRITO, M. F. P; NETTO, W. F. S.; MIRANDA, M. V. F. E. S.; JUNIOR, I. A. S.;DANTAS, C. C.; SANTOS, V. A.; MELO, S. B.; LIMA E. A. O. Monitoring catalyst flow rate in a fcc 
cold pilot unity by gamma ray transmission measurements. International Nuclear Atlantic Conference, 24-29, 2013;

[8] PELOWITZ, D. B., MCNPX User's Manual Version 2.6.0, Los Alamos National Laboratory, United States of America, 2008.

[9] NIST - National Institute of Standards and Technology, XCOM. Mass attenuation coefficient. Disponível em < https://physics.nist.gov/cgi-bin/Xcom/xcom2 >. Acesso em: 10 de Agosto de 2019.

[10] FRIEDLANDER, G; KENNEDY, J. W; MACIAS, E. S; MILLER, J. M. Nuclear and radiochemistry. 3. Ed. New York: A wiley-interscience publication, 1981.

[11] SHULTIS, J. K.; FAW, R. E. An monp primer. 2011. Disponível em: <http://www.mne.kstate.edu/ jks/MCNPprmr.pdf>. Acesso em: 10 dez. 2019.

[12] AZZI, M.; TURLIER, J.; BERNARD, R.; GARNERO, L. Mapping solid concentration in a circulating fluid bed using gammametry. Powder Technology. Vol. 67, p. 27-36, 1991. 